\title{
Examining microcavity organic light-emitting devices having two metal mirrors
}

\author{
Chun-Liang Lin, Hao-Wu Lin, and Chung-Chih $\mathrm{Wu}^{\mathrm{a})}$ \\ Department of Electrical Engineering, Graduate Institute of Electro-optical Engineering, and Graduate \\ Institute of Electronics Engineering, National Taiwan University, Taipei, Taiwan 10617, Republic \\ of China
}

(Received 5 January 2005; accepted 25 May 2005; published online 5 July 2005)

\begin{abstract}
Optical characteristics of microcavity organic light-emitting devices (OLEDs) having two metal mirrors are examined. Analyses show that a high-reflection back mirror and a low-loss high-reflection exit mirror are essential for such microcavity devices to obtain luminance enhancement relative to conventional noncavity devices. An enhancement of $2 \mathrm{in} \mathrm{cd/A} \mathrm{efficiencies}$ has been experimentally achieved for microcavity top-emitting OLEDs using an exit mirror composing thin metal and dielectric capping. The capping layer in the composite mirror plays the role of enhancing reflection and reducing absorption loss, rather than enhancing transmission.
\end{abstract}

(C) 2005 American Institute of Physics. [DOI: 10.1063/1.1988985]

Incorporation of the microcavity structure into organic light-emitting devices (OLEDs) is often demonstrated to narrow emission and improve color purity for displays. ${ }^{1-7}$ Using lossless dielectric mirrors, microcavity OLEDs give substantially enhanced luminance in comparison with conventional noncavity OLEDs. ${ }^{2,3}$ General complexity of dielectric mirrors, however, render difficult their implementation into displays. In view of these, microcavity OLEDs using metal mirrors are more practical for displays. ${ }^{1,5-7}$ However, due to absorption (loss) in metals, it is not clear to what degree and under what conditions one obtains most luminance enhancement from such microcavity OLEDs. In this letter, we examine optical characteristics of microcavity OLEDs having two metal mirrors.

Microcavity OLEDs may be considered as a FabryPerot cavity embedded with a source as shown in Fig. 1, in which $E_{0}$ represents the free-space electric-field intensity of the source, $E_{2}$ is the electric-field intensity of the outcoupled wave, $\sqrt{ } R_{1} e^{j \phi 1}$ is the complex reflection coefficient of the back mirror $\mathrm{M}_{1}$ (with a reflectance $R_{1}$ ), ${ }_{\sqrt{2}} e^{j \phi 2}$ is the complex reflection coefficient of the exit mirror $\mathrm{M}_{2}$ (with a reflectance, a transmittance, and an absorptance of $R_{2}, T_{2}$, and $A_{2}$, respectively), $L_{1}$ is the source-to- $\mathrm{M}_{1}$ distance, $L_{2}$ is the source-to- $\mathrm{M}_{2}$ distance, and $L$ is the total cavity length ( $L$ $\left.=L_{1}+L_{2}\right)$. Considering both the field redistribution due to cavity and the influence of cavity on the transition rate of molecular excited states, one obtains the emission enhancement factor $G_{\text {cav }}(\lambda)$ (in the forward direction) relative to free-space emission at a wavelength $\lambda:^{2,3,8}$

$G_{\text {cav }}(\lambda)=\frac{\left|E_{2}\right|^{2}}{\left|E_{0}\right|^{2}} \times \frac{\tau_{\text {cav }}}{\tau_{0}}=\left[1-\frac{4 \sqrt{R_{1}} \sin ^{2}\left(\frac{\phi_{1}-2 k L_{1}}{2}\right)}{\left(1+\sqrt{R_{1}}\right)^{2}}\right]$

${ }^{a}$ Author to whom correspondence should be addressed; electronic mail: chungwu@cc.ee.ntu.edu.tw

$$
\times\left[\frac{T_{2}\left(1+\sqrt{R_{1}}\right)^{2}}{\left(1-\sqrt{R_{1} R_{2}}\right)^{2}+4 \sqrt{R_{1} R_{2}} \sin ^{2}\left(\frac{\phi_{1}+\phi_{2}-2 k L}{2}\right)}\right] \frac{\tau_{\mathrm{cav}}}{\tau_{0}},
$$

where $k$ is the wave vector in the organic layer, and $\tau_{\text {cav }}$ and $\tau_{0}$ are lifetimes of the molecular excited state in the cavity and in the free space, respectively.

For convenience of analysis, consider an emitter with an intrinsic emission spectrum $S(\lambda)$ which is assumed Gaussian with a peak wavelength $\lambda_{\mathrm{em}}$ and a full width at half maximum (FWHM) $\Delta \lambda_{\mathrm{em}}$. According to Eq. (1), to maximize luminance from a cavity with a particular pair of mirrors, one must set the resonance $\left(\phi_{1}+\phi_{2}-2 k L=2 m \pi, m\right.$ : Integer $)$ near $\lambda_{\mathrm{em}}$ and place the emitter near the antinode of $\mathrm{M}_{1}\left(\phi_{1}\right.$ $-2 k L_{1}=2 \ell \pi$, $\ell$ : integer). $G_{\text {cav }}(\lambda)$ in Eq. (1) describes the single-wavelength enhancement (in the forward direction). Of more practical interest, however, is the spectrally integrated enhancement $G_{\text {int }}$ of an optimized microcavity device, as defined in Eq. (2), relative to emission from a conventional noncavity (indeed weak-microcavity) bottom-emitting OLED that has a indium tin oxide (ITO) as the bottom electrode and a high-reflectivity top metal electrode:

$$
G_{\text {int }}=\int S(\lambda) G_{\text {cav }}(\lambda) d \lambda / \int S(\lambda) G_{\text {con }}(\lambda) d \lambda,
$$

where $G_{\text {con }}(\lambda)$ is the emission enhancement of an optimized noncavity OLED (i.e., satisfy antinode and resonance conditions) as described in Eq. (1). For such a conventional OLED, $R_{1} \sim 90 \%$ (from $\mathrm{Al}$ or $\mathrm{Ag}$ electrodes) and $R_{2} \sim 3 \%$ (from the ITO/glass interface) are assumed.

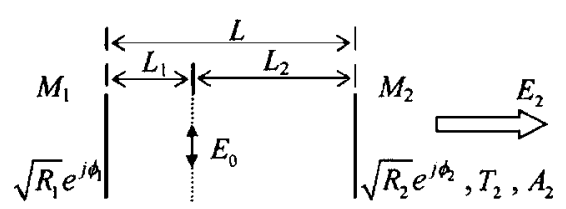

FIG. 1. Schematic diagram showing the structure and characteristics of a microcavity device. 

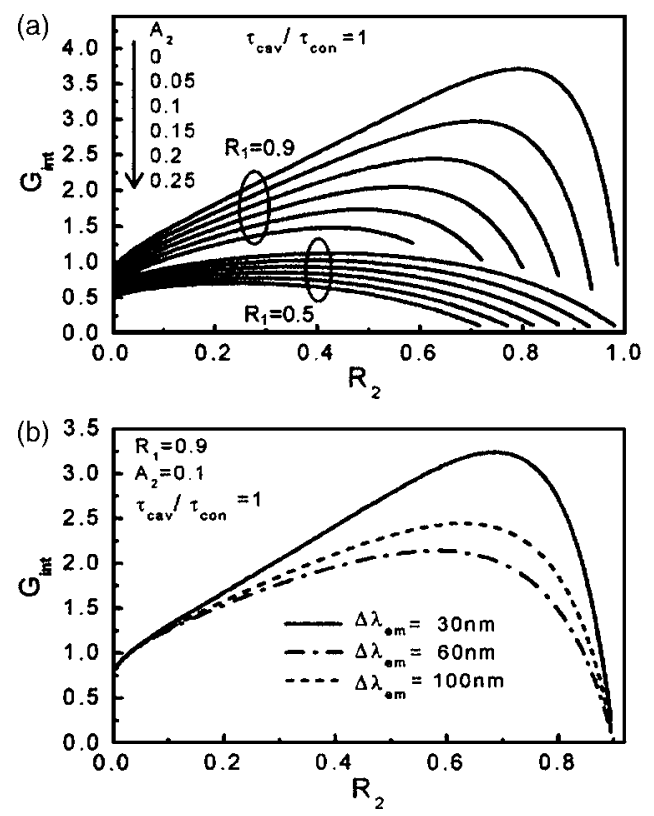

FIG. 2. Calculated $G_{\text {int }}$ as a function of $R_{2}\left(\lambda_{\mathrm{em}}=520 \mathrm{~nm}\right)$ : (a) $\Delta \lambda_{\mathrm{em}}$ $=60 \mathrm{~nm}$; (b) $\Delta \lambda_{\mathrm{em}}$ varied.

In general, $G_{\text {int }}$ is a function of $R_{1}, R_{2}, A_{2}\left(T_{2}=1-R_{2}\right.$ $-A_{2}$ ), and $\Delta \lambda_{\text {em }}$ according to Eqs. (1) and (2). Since $\tau_{\text {con }}$, the molecular excited-state lifetime in the conventional noncavity device in general differs from $\tau_{\text {cav }}, G_{\text {int }}$ also depends on the ratio $\tau_{\text {cav }} / \tau_{\text {con }}$. Experimentally measured $\tau_{\text {cav }} / \tau_{\text {con }}$, however, is usually close to $1 .^{2,3,9,10}$ Thus, for a temporary purpose, it is set to 1 for first examining influences of other parameters. Figure 2(a) shows calculated $G_{\text {int }}$ for the lowest cavity mode $(m=0, \ell=0)$ as a function of $R_{2}$ with $\Delta \lambda_{\mathrm{em}}$ $=60 \mathrm{~nm}$ (and $\lambda_{\mathrm{em}}=520 \mathrm{~nm}$ ), $R_{1}=0.9-0.5$ and $A_{2}=0-0.25$. Results of Fig. 2(a) indicate that with $R_{1}$ large enough and $A_{2}$ small enough, one observes $G_{\text {int }}>1$ as long as M2 $\left(R_{2}\right)$ is carefully designed. $G_{\text {int }}$ drops rapidly with the decrease of $R_{1}$ and the increase of $A_{2}$. In the ideal (lossless) case of $A_{2}=0$, $G_{\text {int }}$ as high as $\sim 4$ may be obtained. Figure 2(b) shows that narrower molecular emission spectra better match the peak region of $G_{\text {cav }}(\lambda)$ [FWHM $\sim 51 \mathrm{~nm}$ in this case], and thus give larger enhancement. In Fig. 2, of particular interest is the value of $R_{2}$ for achieving maximal $G_{\text {int }}$ in each curve. It can be quickly estimated with $R_{2}=R_{1}\left(1-A_{2}\right)^{2}$, which maximizes $G_{\text {cav }}\left(\lambda_{\text {em }}\right) / G_{\text {con }}\left(\lambda_{\text {em }}\right)$. True optimal $R_{2}$ is slightly lower than this value, so that FWHM of $G_{\text {cav }}(\lambda)$ expands to better match $\Delta \lambda_{\mathrm{em}}$.

Based on preceding analyses, top-emitting OLEDs (TOLEDs), ${ }^{5-7,11-14}$ usually showing stronger microcavity effects are then examined. A TOLED usually contains a reflective bottom electrode (M1) and a (semi)transparent top electrode (M2) for light outcoupling. To get maximal luminance from TOLEDs, the highest possible $R_{1}$ (bottom electrode) and a $R_{2}$ (top electrode) matching $R_{1}$ should be adopted. In view of this, making the top electrode as transparent as possible (i.e., $R_{2} \rightarrow 0$ ), as thought in early TOLED developments, $5,11-14$ gives luminance only roughly as much as conventional bottom-emitting OLEDs. Fully transparent electrodes usually also have issues of high sheet resistance. On the other hand, using semitransparent metals as the top electrodes provide lower sheet resistance for current conduc-

tion and stronger reflection required.
Downloaded $17 \mathrm{Feb} 2009$ to 140.112 .113 .225 . Redistribution subject to AlP license or copyright; see http://apl.aip.org/apl/copyright.jsp
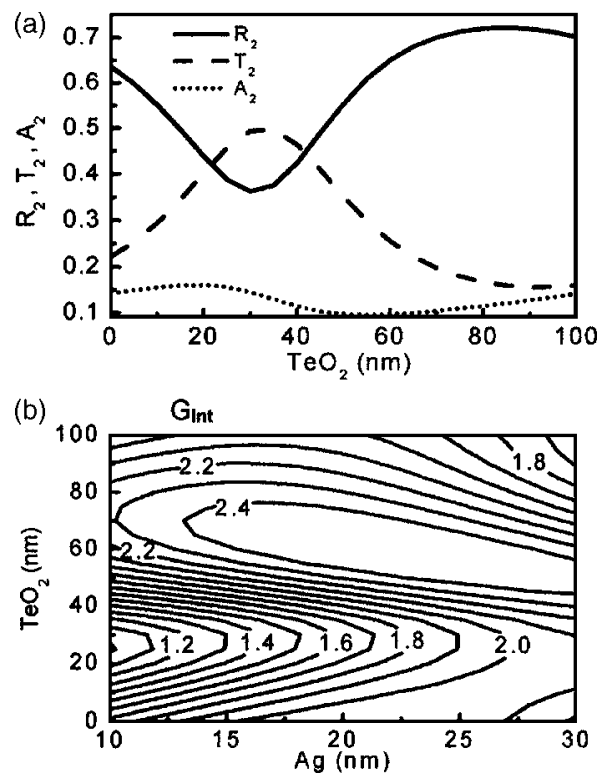

FIG. 3. (a) Calculated $R_{2}, T_{2}, A_{2}$ (at $520 \mathrm{~nm}$ ) of thin $\mathrm{Ag}(24 \mathrm{~nm}$ ) capped with $\mathrm{TeO}_{2}$. (b) Contour plot of calculated $G_{\text {int }}$ vs $\mathrm{Ag}$ and $\mathrm{TeO}_{2}$ thicknesses for $R_{1}=0.9, \lambda_{\mathrm{em}}=520 \mathrm{~nm}, \Delta \lambda_{\mathrm{em}}=60 \mathrm{~nm}, \tau_{\mathrm{cav}} / \tau_{\mathrm{con}}=1$.

For a semitransparent metal electrode, at the thickness of large enough $R_{2}$, the absorptance $A_{2}$, however, may become too large and degrade luminance enhancement. The top electrode structure combining a thin metal and a transparent capping layer (either dielectric, semiconductive or conductive) overcomes such a dilemma. Figure 3(a) shows the calculated $R_{2}, T_{2}$, and $A_{2}$ at $520 \mathrm{~nm}$ viewed from a typical organic material for a thin $\mathrm{Ag}$ electrode $(24 \mathrm{~nm})$ capped with a highindex $\mathrm{TeO}_{2}(n \sim 2.2-2.3)$ of varied thickness. ${ }^{7} \mathrm{Ag}$ is considered as it has relatively larger reflection, smaller absorption and higher conductivity among metals. $R_{2}$ is largely modulated with the $\mathrm{TeO}_{2}$ thickness, and for a certain range of thickness, $R_{2}$ is enhanced and $A_{2}$ is reduced down to $\sim 9 \%$ (versus $15 \%$ with no capping). Capping layers with higher indices usually have the advantage of giving stronger modulation in optical characteristics and thus possibly higher luminance enhancement. To search for combinations of $\mathrm{Ag} / \mathrm{TeO}_{2}$ giving optimal luminance enhancement, it is useful to construct a contour plot of $G_{\text {int }}$ versus $\mathrm{Ag}$ and $\mathrm{TeO}_{2}$ thicknesses [Fig. 3(b)]. For a certain range of $\mathrm{Ag} / \mathrm{TeO}_{2}$ combinations, one obtains maximal $G_{\text {int }}$ of $\sim 2.4-2.6$. Detailed analyses reveal that these conditions are roughly coincident with those giving high reflection and low absorption. Such findings are in contrast with one previous viewpoint that improving light outcoupling from a thin metal electrode with transparent capping is due to enhanced transmission. 6,7,14

Experiments were conducted on microcavity TOLEDs with a nearly optimized structure of: glass/Ag $(80 \mathrm{~nm}) / \mathrm{m}$-MTDATA:F4-TCNQ $\quad(2 \mathrm{wt} \%, 20 \mathrm{~nm}) / \alpha$-NPD $(25 \mathrm{~nm}) / \mathrm{Alq}_{3}: \mathrm{C} 545 \mathrm{~T}(1 \mathrm{wt} \%, 20 \mathrm{~nm}) / \mathrm{Alq}_{3}(35 \mathrm{~nm}) / \mathrm{LiF}$ $(0.5 \mathrm{~nm}) / \mathrm{Al}(1 \mathrm{~nm}) / \mathrm{Ag}(24 \mathrm{~nm}) / \mathrm{TeO}_{2}(55 \mathrm{~nm}) . \mathrm{Ag}$ and $\mathrm{Ag} / \mathrm{TeO}_{2}$ serve as the bottom anode (M1) and the top cathode (M2), respectively. Other layers consist of 4, 4', 4"-tris(3-methylphenylphenylamino)triphenylamine (m-MTDATA) doped with 2 wt \% of tetrafluorotetracyanoquinodimethane (F4-TCNQ) as the hole-injection layer, ${ }^{15}$ $\alpha$-naphthylphenylbiphenyl diamine ( $\alpha$-NPD) as the holetransport layer, ${ }^{14}$ tris-(8-hydroxyquinoline) aluminum $\left(\mathrm{Alq}_{3}\right)$ doped with the fluorescent dye $\operatorname{C545T}\left(\Delta \lambda_{\text {em }} \approx 60 \mathrm{~nm}\right)$ as the
to AlP license or copyright; see http://apl.aip.org/apl/copyright.jsp 


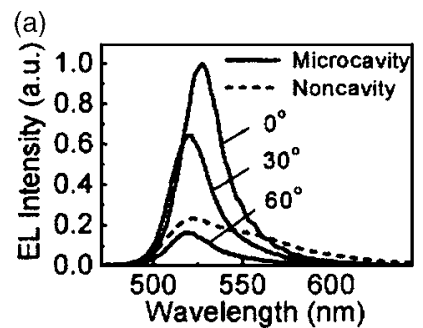

(b)
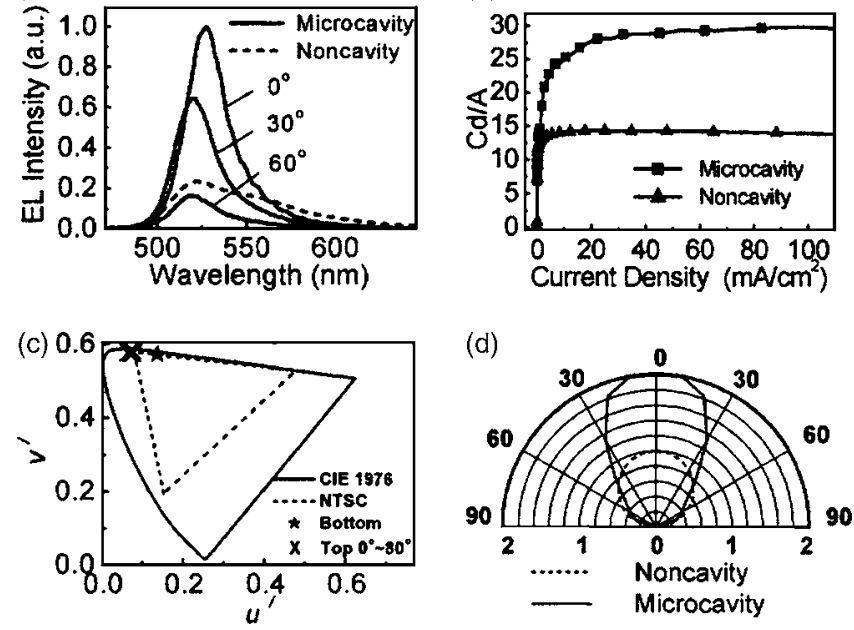

FIG. 4. (a) Measured EL spectra with relative intensities at $0^{\circ}, 30^{\circ}$, and $60^{\circ}$ off the surface normal of the microcavity device, and at $0^{\circ}$ of the noncavity device. (b) cd/A efficiencies for both devices. (c) 1976 CIE coordinates of $0^{\circ}-80^{\circ} \mathrm{EL}$ of the microcavity device and $0^{\circ} \mathrm{EL}$ of the noncavity device. (d) Polar plots of measured EL intensities (normalized to the $0^{\circ}$ intensity of the noncavity device) for both devices.

emitting layer, undoped $\mathrm{Alq}_{3}$ as the electron-transport layer, ${ }^{14}$ and thin LiF/ Al as the electron injection layer. ${ }^{14}$ For comparison, an optimized noncavity bottom-emitting device was also fabricated with the structure of: glass/ITO $(120 \mathrm{~nm}) / \mathrm{m}$-MTDATA:F4-TCNQ (2 wt \% , $20 \mathrm{~nm}) / \alpha$-NPD $(20 \mathrm{~nm}) / \mathrm{Alq}_{3}:$ C545T $(1 \mathrm{wt} \%, 20 \mathrm{~nm}) / \mathrm{Alq}_{3} \quad(40 \mathrm{~nm}) / \mathrm{LiF}$ $(0.5 \mathrm{~nm}) / \mathrm{Al}(1 \mathrm{~nm}) / \mathrm{Ag}(150 \mathrm{~nm})$. In both devices, layer thicknesses had been determined based on the antinode and resonance conditions near $\lambda_{\mathrm{em}}$ of C545T (520 nm).

Figure 4(a) shows the measured electroluminescence (EL) spectra with relative intensities at viewing angles of $0^{\circ}$, $30^{\circ}$, and $60^{\circ}$ off the surface normal of the microcavity device, and at $0^{\circ}$ of the noncavity device. The microcavity device shows a forward $G_{\text {int }}$ of $\sim 2.0$ (and an enhancement of $\sim 4.3$ at the $0^{\circ}$ resonance wavelength). Correspondingly, the microcavity device gives a $\sim 2.0$ times larger $\mathrm{cd} / \mathrm{A}$ efficiency than the noncavity device [30 cd/A versus $14.2 \mathrm{~cd} / \mathrm{A}$, Fig. 4(b)]. Figure 4(c) shows the CIE coordinates (1976 uniform color space) of $0^{\circ}-80^{\circ} \mathrm{EL}$ of the microcavity device and that of the noncavity device. The microcavity device shows more saturated colors than the NTSC green standard and negligible color shift with viewing angles, both the desired characteristics for displays. Figure 4(d) compares the angular distributions of EL intensity (normalized to the $0^{\circ}$ intensity of the noncavity device) for both devices. The microcavity device shows more directed emission and enhanced luminance for $0^{\circ}-45^{\circ}$. Such characteristics shall be an advantage for small- or medium-sized OLED displays, which are mainly viewed from the forward direction. The external EL quantum efficiency for the cavity device is slightly higher than that of the noncavity device $(4.1 \%$ versus $3.8 \%)$, indicating the forward-direction enhancement results mainly from redistribution of emission directions.

Luminance enhancement of $\sim 2.0$ is lower than the expected value $[$ i.e., $\sim 2.5$, Fig. $3(\mathrm{~b})]$ assuming $\tau_{\text {cav }} / \tau_{\text {con }} \approx 1$. We have measured excited-state lifetimes of C545T in the

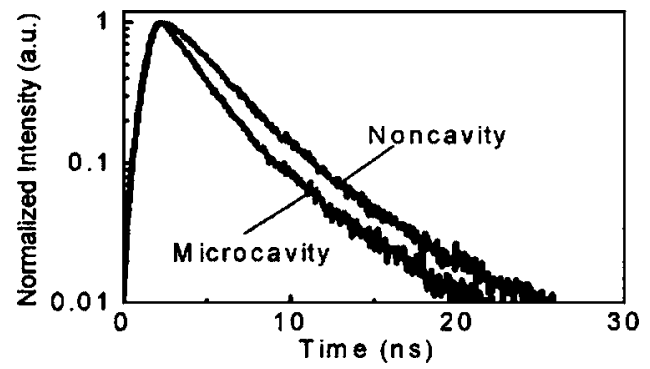

FIG. 5. Photoluminescence decay curves detected at $520 \mathrm{~nm}$ for microcavity and noncavity structures.

microcavity device and the noncavity device using the timecorrelated single-photon counting technique. Figure 5 shows the photoluminescence decay curves detected at $520 \mathrm{~nm}$ for both structures. The microcavity structure shows a faster decay $\left(\tau_{\text {cav }} \sim 2.6 \mathrm{~ns}\right)$ than the noncavity structure $\left(\tau_{\text {con }}\right.$ $\sim 3.2 \mathrm{~ns}$ ), indicating the molecular transition rate be enhanced in the optimized microcavity structure. Together with $G_{\text {int }}$ of $\sim 2.5$ calculated with $\tau_{\text {cav }} / \tau_{\text {con }}=1$, the experimentally determined $\tau_{\text {cav }} / \tau_{\text {con }} \sim 0.8$ gives true $G_{\text {int }}$ of $\sim 2.0$, consistent with the measured value.

In summary, we have examined emission characteristics of microcavity OLEDs having two metal mirrors. General conditions for obtaining luminance enhancement from such cavity devices are established. Even higher enhancement may be realized with clever inventions of emitting materials with narrower emission and high-reflection mirrors with even lower absorption.

The authors would like to acknowledge financial support from National Science Council of Republic of China and Aixtron Co. One of the authors (C.L.L.) is also grateful for financial support from MediaTek Fellowship.

${ }^{1}$ N. Takada, T. Tsutsui, and S. Saito, Appl. Phys. Lett. 63, 2032 (1993).

${ }^{2}$ A. Dodabalapur, L. J. Rothberg, R. H. Jordan, T. M. Miller, R. E. Slusher, and J. M. Phillips, J. Appl. Phys. 80, 6954 (1996).

${ }^{3}$ R. H. Jordan, L. J. Rothberg, A. Dodabalapur, and R. E. Slusher, Appl. Phys. Lett. 69, 1997 (1996)

${ }^{4}$ S. Tokito, K. Noda, and Y. Taga, Appl. Phys. Lett. 68, 2633 (1996).

${ }^{5}$ M.-H. Lu, M. S. Weaver, T. X. Zhou, M. Rothman, R. C. Kwong, M. Hack, and J. J. Brown, Appl. Phys. Lett. 81, 3921 (2002).

${ }^{6}$ H. Riel, S. Karg, T. Beierlein, W. Rieß, and K. Neyts, J. Appl. Phys. 94, 5290 (2003).

${ }^{7}$ C.-W. Chen, P.-Y. Hsieh, H.-H. Chiang, C.-L. Lin, H.-M. Wu, and C.-C. Wu, Appl. Phys. Lett. 83, 5127 (2003).

${ }^{8}$ E. F. Schubert, N. E. J. Hunt, M. Micovic, R. J. Malik, D. L. Sivco, A. Y. Cho, and G. J. Zydzik, Science 265, 943 (1994).

${ }^{9}$ G. R. Hayes, F. Cacialli, and T. R. Phillips, Phys. Rev. B 56, 4798 (1997).

${ }^{10}$ U. Lemmer, R. Hennig, W. Guss, A. Ochse, J. Pommerehne, R. Sander, A. Greiner, R. F. Mahrt, H. Bässler, J. Feldmann, and E. O. Göbel, Appl. Phys. Lett. 66, 1301 (1995).

${ }^{11}$ T. Sasaoka, M. Sekiya, A. Yumoto, J. Yamada, T. Hirano, Y. Iwase, T. Yamada, T. Ishibashi, T. Mori, M. Asano, S. Tamura, and T. Urabe, 2001 Society for Information Display (SID) International Symposium, Digest of Technical Papers (San Jose, CA, 2001), p. 384.

${ }^{12}$ G. Gu, V. Bulovic, P. E. Burrows, S. R. Forrest, and M. E. Thompson, Appl. Phys. Lett. 68, 2006 (1996).

${ }^{13}$ G. Parthasarathy, P. E. Burrows, V. Khalfin, V. G. Kozlov, and S. R. Forrest, Appl. Phys. Lett. 72, 2138 (1998).

${ }^{14}$ L. S. Hung, C. W. Tang, M. G. Mason, P. Raychaudhuri, and J. Madathil, Appl. Phys. Lett. 78, 544 (2001).

${ }^{15}$ X. Zhou, M. Pfeiffer, J. Blochwitz, A. Werner, A. Nollau, T. Fritz, and K. Leo, Appl. Phys. Lett. 78, 410 (2001). 